\title{
Establecimiento de indicadores de sostenibilidad para entornos degradados: el Valle minero de Laciana (León, España)
}

\author{
Settings for sustainability indicators in mining landscapes: \\ the mining Valley of Laciana (León, Spain)
}

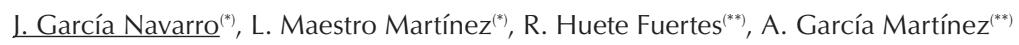

\section{RESUMEN}

La interacción entre los edificios, el medio ambiente y su entorno socioeconómico, así como los impactos que resultan de la misma es algo tan complejo como el propio proceso constructivo. Un análisis riguroso de las afecciones producidas por la actividad edificatoria debe tener en cuenta el ciclo de vida completo, tanto del edificio como de sus productos y componentes. En cada fase, el impacto se produce en todos los niveles; desde un nivel local, como por ejemplo la huella que produce una cantera, a un nivel global, como las emisiones de dióxido de carbono producido por el consumo de combustible para el acondicionamiento de los recintos. Por ello, para un mismo impacto, se trabaja con diferentes escalas.

El establecimiento de un sistema de indicadores se ha configurado en los últimos años como un método de evaluación común de un producto, proceso o actividad. No obstante, el proceso constructivo presenta singularidades que es necesario considerar.

En el marco del proyecto de investigación del Plan Nacional I+D+i 2004-2007 "Modelo de construcción sostenible para la recuperación y protección de zonas medioambientalmente degradadas o frágiles: el Valle de Laciana" (BIA 2004-7654), se ha desarrollado un modelo de intervención para construcciones, infraestructuras y su entorno, desde criterios de sostenibilidad. En este artículo, se exponen los resultados alcanzados sobre la aplicabilidad de indicadores y las consideraciones a tener en cuenta en su selección para la evaluación de elementos en general, $y$ de edificios en particular.

113-83

Palabras clave: construcción, sostenibilidad, indicadores, paisaje minero.

\section{SUMMARY}

The interaction among building, environment, and socioeconomic matters, results in impacts as complex as the building process itself. A straight analysis of these impacts or effects must consider the whole building life-cycle, building products and building components. Also impacts are found in every level; from a local level, for example, the fingerprints a quarry leaves on the landscape, to a global level, i.e. $\mathrm{CO}_{2}$ emissions due to energy consumption used to reach wealthy indoor conditions. Every impact must be considered differently in every level and phase.

In the last years, indicator sets are drawn as appropriate tools in assessment methods for products, processes or activities. Nevertheless, the building process involves other singularities to be considered.

Within the framework of the R\&D National Plan Program, the project titled "Model of sustainable construction for the rehabilitation and protection of environmentally degraded or fragile zones: the Mining Valley of Laciana" (García Navarro et al., 2005-2007) has developed a model following the sustainability criteria in construction, for buildings, existing infrastructures and landscapes in mining regions. This paper analyzes the application of indicator sets in this type of scenarios, and provides practical recommendations in order to develop integrating models or guidelines in the assessment, planning and management of these interventions.

Keywords: building, sustainability, indicators, mining landscape.
Informes de la Construcción Vol. 61, 514, 51-70, abril-junio 2009 ISSN: 0020-0883 elSSN: $1988-3234$ doi: $10.3989 /$ ic.08.022

\footnotetext{
(*) Universidad Politécnica de Madrid. E.T.S.I.A. (Madrid, España)

$(* *)$ Universidad de Sevilla. E.T.S.A.S. (Sevilla, España)

Persona de contacto/Corresponding author: justo.gnavarro@upm.es (J. García Navarro)
}

Fecha de recepción: 03-07-08 Fecha de aceptación: 22-01-09 


\section{INTRODUCCIÓN}

El establecimiento de un sistema de indicadores se ha configurado en los últimos años como un método de evaluación común de un producto, proceso o actividad. No obstante, el proceso constructivo presenta singularidades que es necesario considerar.

La interacción entre los edificios, el medio ambiente y su entorno socioeconómico, así como los impactos que resultan de esta interacción es algo tan complejo como el propio proceso constructivo. Un análisis riguroso de las afecciones producidas por la actividad edificatoria debe tener en cuenta el ciclo de vida completo, tanto del edificio como de sus productos y componentes. En cada fase, el impacto se produce en todos los niveles; desde un nivel local, como por ejemplo la huella que produce una cantera, hasta un nivel global, como las emisiones de dióxido de carbono a la atmósfera producidas por el consumo de combustible para el acondicionamiento de los recintos. Por ello, para un mismo impacto, se trabaja con diferentes escalas.

La proliferación de iniciativas de las administraciones, organizaciones no gubernamentales y empresas privadas, a favor del desarroIlo sostenible, ha multiplicado y complicado extraordinariamente el entendimiento (5) y la definición de indicadores, que sin embargo, se han configurado como una herramienta eficaz para el análisis de tendencias y la propuesta de objetivos.

\subsection{Consideraciones generales de los requerimientos de un indicador}

Los indicadores son medidas cuantitativas, cualitativas o descriptivas (10-12), que permiten simplificar la información disponible acerca de un elemento y/o de la calidad de un proceso (incluyendo desarrollo, planificación, preparación y operación), en una forma relativamente sencilla de utilizar y de comprender.

Las tres funciones principales de los indicadores son la cuantificación, la simplificación y la comunicación. La variación de los cambios en el tiempo y el desarrollo de cambios con relación a los objetivos preestablecidos, pueden monitorizarse con ayuda de los indicadores. Una de las funciones más importantes de un indicador, en referencia a la toma de decisiones, es su potencial para mostrar una tendencia: los indicadores deben ser objetivos y sus resultados deben ser reproducibles (13).

En una fase posterior de análisis de los resultados de los indicadores, pueden establecerse índices. Los índices son valores que se construyen agregando diversas variables que se asumen como componentes de un fenómeno y a las cuales se les asigna un peso relativo con respecto al resto a la hora de sumar todos los efectos (18). Los índices, por lo tanto, permiten la composición de una "foto fija" representativa de todo el fenómeno, que nos ayudan a analizar tendencias y establecer objetivos. Ejemplos de índices relacionados con el Desarrollo Sostenible son el Índice de Bienestar Económico Sostenible (IBES), el índice de sostenibilidad Ambiental (ISA), la huella ecológica, o el índice del Planeta Vivo (Word Wildlife Fund International). Deben tener consistencia en las áreas del mismo contexto. Esto se ve favorecido cuando la información es uniforme.

\subsection{Caso particular: los indicadores en el sector de la construcción}

La selección de indicadores está condicionada por las inquietudes de los agentes implicados en el contexto de la actuación y la apropiada representación del elemento en evaluación. Cuando se eligen y desarrollan indicadores, el punto de partida es la identificación de los principales usuarios y sus necesidades, ya que la escala espacio-temporal influye en la elección de los indicadores con mayor relevancia (22). Los indicadores son necesarios en la toma de decisiones de:

- Empresas, Promotores y Propietarios de los edificios y sitios

- Proyectistas, Diseñadores, Investigadores

- Administración

- Usuarios

- Gestores de mantenimiento del patrimonio

Para el uso efectivo de los indicadores, es necesario:

- Elegir los indicadores relevantes

- Encontrar los métodos adecuados y la información para evaluar sus valores

La elección de los indicadores relevantes dependerá de las necesidades de las partes interesadas, los límites de decisión, las fases de aplicación del objeto (el contexto del elemento), y la disponibilidad de información. En el proceso de selección de un grupo estructurado de indicadores (3) destacan los siguientes requerimientos:

- No debe haber un elevado número de indicadores. Un número reducido de indicadores debe ser suficiente para reflejar las tendencias más importantes o de interés en un aspecto concreto del caso de estudio;

- Suelen ser preferibles los indicadores de carácter cuantitativo;

- Los indicadores deben tener un cierto grado de sensibilidad; 
- Deben ser simples de interpretar por los diferentes usuarios para su utilidad en la toma de decisiones.

La otra etapa es la recopilación de información y el uso de los métodos relevantes con el fin de asignar valores a los indicadores seleccionados.

Como los indicadores pueden usarse para simplificar y dar información compleja, pueden ser útiles en los siguientes campos de aplicación:

- Evaluación (ej.: contra valores objetivo establecidos)

- Diagnóstico (ej.: señalar factores afectados)

- Comparación (ej.: con edificios de referencia)

- Seguimiento (ej.: cambios en el tiempo) y Control

Los indicadores deben reflejar y considerar aquellos aspectos que permiten expresar o describir la adecuación o sostenibilidad que presentan los elementos.

El carácter, disponibilidad y especificidad de la información varía según la etapa de vida en la que se encuentre el elemento, los condicionantes inherentes y factores externos. Es lógico pensar, que durante la etapa de funcionamiento existirá una mayor información disponible sobre el comportamiento del elemento.

Por ello, los indicadores que tratan una misma cuestión pueden relacionarse inicialmente con valores pronosticados durante la etapa de diseño, mientras que durante la operación, estos indicadores pueden estar basados en medidas, muestreos, controles, etc.

Las directrices y pautas para la selección de materiales, productos, sistemas o procesos deberán considerarse como recomendaciones prácticas, pero no como indicadores propiamente dichos. Estas recomendaciones pueden evaluarse posteriormente con la ayuda de indicadores de sostenibilidad. Las recomendaciones prácticas, que favorecen ciertos tipos de soluciones técnicas, pueden depender de las circunstancias climatológicas, geográficas y tecnológicas. Los indicadores son más genéricos, aunque los valores aceptables para cada indicador vendrán dados por el emplazamiento.

Una fase necesaria para el desarrollo de indicadores efectivos es la formulación de una terminología apropiada y un marco de trabajo en el que se establezcan detalles científicos y técnicos. Deben trabajar en tres ámbitos diferenciados, para satisfacer las ne- cesidades políticas europeas, nacionales, y específicas locales.

Por último, la monitorización de los datos permite establecer la presencia o ausencia de tendencias (16).

\section{MARCO PARA LOS INDICADORES DE SOSTENIBILIDAD ${ }^{1}$}

En el contexto del desarrollo sostenible, se hablará de indicadores de sostenibilidad, entendiendo que son aquellos que engloban aspectos $^{2}$ económicos, medioambientales y sociales $^{3}$ y sus interrelaciones. El ámbito de actuación de estos indicadores ha sido definido y desarrollado en proyectos de investigación y desde organismos internacionales de muy diversa índole. En algunos casos, pretenden sentar una base normativa y de consenso internacional, lo que les confiere una mayor confianza en relación con su validez (6).

La elección de un sistema estructurado de indicadores resulta relevante para:

- Describir un estado y predecir efectos de sistemas complejos;

- Comparar alternativas y sistematizar el seguimiento;

- Proporcionar un cuadro representativo de las condiciones medioambientales o socioeconómicas de un territorio, comparables en otros ámbitos regionales, nacionales e internacionales.

En los casos en los que no se pueda ofrecer un indicador, se dará la oportuna aproximación, ya sea en forma de recomendación o directriz para la selección y el desarrollo de indicadores apropiados para definir el proceso que debe seguirse cuando se trata el impacto ambiental, social y económico de un elemento. Con todo ello se pretende:

- Adaptar los principios generales de sostenibilidad a cada elemento, para establecer un marco para la evaluación;

- Mostrar indicadores como ejemplo;

- Mostrar cómo usar indicadores de sostenibilidad respecto a estos elementos, y también el proceso de uso de indicadores de sostenibilidad;

- Apoyar el proceso de elección de indicadores y de otras herramientas de evaluación.

Cuando se trabaja en alguna de las dimensiones específicas del desarrollo sostenible, se utilizan consecuentemente los correspondientes indicadores ambientales, económicos o sociales, variables que independientemente de su dimensión, reflejan implícitamente una preocupación social por el medio ambiente, el desarrollo económico o el bienestar. Esta
${ }^{1}$ Este trabajo ha tomado como una de sus primeras referencias, la norma ISO 21929. 1:2006 [en español UNE-ISO/ TS 21929-1:2009 IN], en cuya redacción han trabajado varios de los autores.

${ }^{2}$ Un aspecto podrá hacer alusión o referencia a un impacto, ya sea de tipo medioambiental, social o económico, o a cuestiones que tengan consecuencias directas en ese impacto.

${ }^{3}$ Los aspectos culturales quedan englobados dentro del ámbito social. 
preocupación debe transmitirse de forma coherente durante el proceso de toma de decisiones de los propios agentes implicados.

Por tanto, se emplearán:

\subsection{Indicadores ambientales}

Un indicador medioambiental de un elemento trata un aspecto medioambiental, tanto en términos de cargas como de impactos ambientales. Las cargas ambientales consideran el uso de los recursos y la producción de residuos, olores, ruido o emisiones perjudiciales para el suelo, el agua y el aire. Dichas cargas ambientales están relacionadas con impactos ambientales, que pueden expresarse como categorías de impactos ambientales, según especifica la norma ISO 14042 (sección 5.3) ${ }^{4}$. Además de estos dos tipos anteriores, pueden utilizarse indicadores de consecuencia para describir los aspectos medioambientales de un elemento, en este caso con un enfoque hacia aquellos aspectos que influyen en la cantidad de cargas o de impactos. El desarrollo de esta clase de indicadores puede ser necesario, por ejemplo, por su facilidad de uso. Los indicadores de consecuencia deben tener una conexión evidente con las cargas o los impactos ambientales.

Cuando se aplican en el ámbito de la edificación, los indicadores medioambientales deberían considerar, siempre que fuera posible, el análisis desde el punto de vista del ciclo de vida, distinguiendo claramente entre las etapas del ciclo de vida del edificio (diseño, fabricación, construcción, uso y fin de vida); en algunos casos, los impactos del uso, mantenimiento, demolición/ reciclaje y vertido final pueden ser decisivos. Si se pretende establecer indicadores para medir el impacto medioambiental de un edificio para un periodo de tiempo más corto (distinto del ciclo de vida completo), como es el caso de edificios existentes, esta variación debe ser transparente y estar justificada.

Las primeras movilizaciones y denuncias sociales en pro de una mayor preocupación por el medio ambiente justificaron en su momento un estudio y difusión de los indicadores medioambientales, lo que se refleja en los documentos que se han ido desarrollando (2, $4,8)$, y en los que se observa que los indicadores económicos y sociales quedan relegados a una segunda posición. Esto justifica a su vez la dificultad de abordar estos temas por la falta de expertos en el sector de la construcción, de lo que este artículo se hace eco.

\subsection{Indicadores económicos}

Los indicadores económicos indican los flujos económicos asociados a un elemento.
Cuando se trata de un edificio, la evaluación del impacto económico puede basarse en la economía del ciclo de vida del mismo. En ese caso, se tienen que considerar los costes del ciclo de vida, determinados en la inversión, uso, mantenimiento y reconstrucción, así como los ingresos potenciales y el desarrollo del valor durante la vida útil del edificio en estudio.

Desde el planteamiento del ciclo de vida de un edificio, se pueden establecer los siguientes flujos económicos:

Inversiones: localización, diseño, manufactura de los productos, construcción

- Uso: consumo de energía y de agua, gestión de residuos, etc.

- Mantenimiento y reparación

- Deconstrucción y gestión/ tratamiento de los residuos finales

- Desarrollo del valor económico del edificio

- Rentas o ingresos generados por el edificio y sus servicios ${ }^{5}$

Los indicadores económicos de los edificios deberán proporcionar un equilibrio entre los aspectos económicos a largo y a corto plazo. Cuando el indicador económico considera un periodo de tiempo menor que el ciclo de vida, esto deberá ser transparente y estar adecuadamente justificado. Como en el caso de los indicadores ambientales, los indicadores económicos pueden también ser de consecuencia. Los indicadores económicos de consecuencia deberían indicar el costo del ciclo de vida o la economía del ciclo de vida del edificio en estudio. Estos indicadores están basados en la asunción de que ciertas características del edificio indican ventajas o costes económicos para los propietarios o usuarios del edificio en una escala de tiempo amplia (13).

\subsection{Indicadores Sociales}

Los indicadores sociales se utilizan para describir cómo los elementos interactúan con aspectos relevantes de la sostenibilidad, principalmente de satisfacción del usuario (bienestar y confort); en el caso de una actividad, se analizan los efectos asociados a la misma (minería, construcción) en términos de salud y seguridad de los usuarios.

Los aspectos sociales pueden estudiarse a escala del elemento (p.e. edificio), o a una escala mayor, de comunidad, como es el caso de la mezcla urbana para la promoción de la cohesión social y la participación, el uso mixto del suelo, etc.

Las cuestiones relacionadas con un proceso también pueden utilizarse para indicar as- 
pectos sociales que afectan a un elemento. Así, en el proceso de construcción, se sugieren como ejemplos de aspectos importantes relacionados con el proceso (13):

- Cooperación con los usuarios de la vivienda y los vecinos

- Capacidad para mantener buenas relaciones con el vecindario y para escuchar las opiniones de los vecinos

- Capacidad para apoyar la cohesión social en el proceso, por ejemplo

- Consideración de los diferentes grupos sociales y culturales de los usuarios y sus necesidades particulares

- Promoción del empleo local

\section{TIPOS DE INDICADORES}

Los indicadores de sostenibilidad pueden desarrollarse y utilizarse por separado o todos juntos para aportar información sobre el estado o tendencias de diferentes aspectos de un elemento. Cuando los aspectos medioambientales, económicos y sociales de los edificios son expresados mediante la ayuda de indicadores, la experiencia demuestra que los sistemas de indicadores son más efectivos que un indicador individual.

Las series de indicadores deben organizarse de modo que permitan la inclusión de una amplia representación de los aspectos de la sostenibilidad a la vez que siguen siendo relevantes para las perspectivas de los agentes implicados.

No existe un sistema normalizado, adaptándose cada sistema de indicadores a los requerimientos y objetivos de cada caso. Algunas organizaciones pioneras en el desarrollo de indicadores ambientales son:

1. La OCDE (Organización para la Cooperación y Desarrollo Económico), que en 1991 publicó el primer conjunto de indicadores (Environmental indicators. A preliminary set. OCDE, París 1991). En 1993 publica el "OCDE Core Set of Environmental Indicators", además de otros trabajos de difusión en relación a indicadores agroambientales, etc., Tabla 1.

La OCDE establece tres tipos de indicadores (17):

- Indicadores de presión (driving force indicators), se centran en las causas de cambio de las condiciones ambientales;

- Indicadores de estado (state indicators), subrayan los efectos de una actividad en el medio ambiente, como impactos sobre el suelo, agua o biodiversidad;

- Indicadores de respuesta (response indicators), cubren las acciones llevadas a cabo para responder a los cambios en el estado del medio ambiente.

2. El Departamento de Coordinación de Política y Desarrollo Sostenible (DPCSD) del Secretariado de la ONU y la División de Estadísticas de la ONU (UNSD), colaboraron en una iniciativa para coordinar un número de actividades en el "Programa de Trabajo en Indicadores de Desarrollo sostenible" (18). El Programa de Trabajo fue iniciado en 1995, produjo un listado de 134 indicadores que fueron publicados en el "Indicators of Sustainable Development. Framework and Methodologies", en 1998. Periódicamente editan en su página web (www.un.org/esa/sustdev) una revisión de los indicadores tratados.

En la última edición revisada, de enero de 2007, se compilan 98 indicadores (de los cuales se marcan 50 como Core Indicadors o indicadores-clave) organizados en 14 temas:

Pobreza- Gobierno- Salud- EducaciónDemografía- Riesgos Naturales- Atmósfera- Tierra- Océanos, playas y costas- Agua dulce- Biodiversidad- Desarrollo económico- Globalización económica- Patrones de consumo y producción.

\section{Tabla 1}

Aspectos para el establecimiento de indicadores medioambientales clave, según una selección extraída de una lista más amplia de la OCDE, sobre los temas generales más relevantes desde el punto de vista medioambiental. LaOCDE publica periódicamentelos valores estadísticos de estos indicadores.

\begin{tabular}{|l|l|}
\hline \multirow{3}{*}{ Aspectos de polución } & Cambio climático \\
& Capa de ozono \\
& Calidad del aire \\
& Generación de residuos \\
& Calidad de agua dulce \\
\hline \multirow{5}{*}{ Recursos naturales } & Recursos y fuentes de agua dulce \\
& Recursos forestales \\
& Recursos piscícolas \\
& Recursos energéticos \\
& Biodiversidad \\
\hline
\end{tabular}

Fuente: Key Environmental Indicators (2004). OECD Environment Directorate Paris, France. 
También desarrollan un proyecto concreto, de propuesta para establecer un listado de indicadores agroambientales (PAIS).

En el ámbito nacional, el Ministerio de Medio Ambiente ha publicado la monografía "Indicadores Ambientales. Una propuesta para España" (1996), en la que se presenta las bases de un sistema español de indicadores. Se proponen un total de 79 indicadores organizados en las siguientes áreas temáticas: Atmósfera, Residuos, Medio Urbano y Recursos Naturales.

Para cada tema ambiental, se proponen tres indicadores relacionados con cada uno de los componentes del marco PER (presión, estado, respuesta), según se muestra en la Tabla 2 (5).

El marco de estos indicadores se ha definido, según las bases de la Evaluación de impacto ambiental (14) y la Evaluación Ambiental Estratégicas (15), que pretenden precisamente la prevención de los impactos imprevistos. Estos documentos legislativos contienen disposiciones que constituyen condiciones suficientes a incorporar en un sistema de indicadores.

En los últimos años se ha producido un avance importante en las compilaciones estadísticas de indicadores de desarrollo sostenible, por parte de diversos organismos, entre los que destacan: a) PNUMA (Programa de las Naciones Unidas para el Medio Ambiente): Perspectivas del Medio Ambiente Mundial (PMAM) (28);

b) EUROSTAT (Oficina estadística de las Comunidades Europeas). La oficina estadística de la Comisión Europea produce datos sobre la Unión Europea y promueve la armonización de los métodos estadísticos de los estados miembros;

c) AEMA (Agencia Europea de Medio Ambiente o European Environmental Agen$c y, E E A):$ Edita publicaciones periódicas, como Environmental Signal 2000 y elabora informes sobre Indicadores, que se establecen dentro del marco FUERZA TRACTORA - PRESIÓN - BASE - IMPACTO - RESPUESTA (19, 20, 22);

d) WRI (World Resources Institute) ha elaborado el programa CAIT (Climate Analysis Indicators Tool), que analiza el cambio climático interpretando un conjunto de indicadores. Asimismo desarrolla el proyecto APEM (Pilot Analysis of Global Ecosystems), donde se analizan los ecosistemas globales, y publica periódicamente su trabajo "Recursos Mundiales" (29);

e) IISD (International Institute for Sustainable Development) realiza una interesante labor de compilación de indicadores de sostenibilidad (26);

Tabla 2

Indicadores de presión, estado y respuesta relacionados con el tema Clima.

\begin{tabular}{|c|c|c|c|}
\hline AMBIENTALES & INDICADOR DE PRESIÓN & INDICADOR DE ESTADO & INDICADOR DE RESPUESTA \\
\hline $\begin{array}{l}\text { Destrucción de la } \\
\text { capa de ozono }\end{array}$ & $\begin{array}{l}\text { Producción/consumo de } \\
\text { CFC }_{\mathrm{s}} \text { y halones }\end{array}$ & Espesor de la capa de $\mathrm{O}_{3}$ & $\begin{array}{l}\text { Recuperación de } \mathrm{CFC}_{\mathrm{s}} \text { y } \\
\text { halones }\end{array}$ \\
\hline $\begin{array}{l}\text { Calentamiento } \\
\text { global }\end{array}$ & $\begin{array}{l}\text { Emisiones de } \mathrm{CO}_{2} \\
\text { Emisiones de } \mathrm{CH}_{4} \\
\text { Índice de potencial de } \\
\text { calentamiento global }\end{array}$ & $\begin{array}{l}\text { Concentración de GEls } \\
\text { Cambio de la temperatura } \\
\text { media de España }\end{array}$ & $\begin{array}{l}\text { Intensidad energética } \\
\text { \% de participación de energías } \\
\text { renovables en energía primaria }\end{array}$ \\
\hline Acidificación & $\begin{array}{l}\text { Emisiones de } \mathrm{SO}_{2} \\
\text { Emisiones de } \mathrm{NO}_{2}\end{array}$ & Ph en precipitación & $\begin{array}{l}\text { Capacidad de instalaciones de } \\
\text { tratamiento de } \mathrm{SO}_{2} \text { en fuentes } \\
\text { fijas } \\
\% \text { del parque de vehículos } \\
\text { rodados dotados de catalizador }\end{array}$ \\
\hline $\begin{array}{l}\text { Contaminación } \\
\text { fotoquímica }\end{array}$ & $\begin{array}{l}\text { Inmisión de } \mathrm{NO}_{2} \text { en } \\
\text { medio urbano }\end{array}$ & $\begin{array}{l}\text { Concentración de } \mathrm{O}_{3} \\
\text { troposférico en medio } \\
\text { periurbano }\end{array}$ & \\
\hline $\begin{array}{l}\text { Contaminación } \\
\text { atmosférica }\end{array}$ & $\begin{array}{l}\text { Emisiones de } \mathrm{SO}_{2} \\
\text { Emisiones de } \mathrm{NO}_{2} \\
\text { Turismos por habitante }\end{array}$ & $\begin{array}{l}\text { Inmisiones de } \mathrm{SO}_{2} \text { respecto a } \\
\text { valores legislados } \\
\text { Inmisiones de } \mathrm{NO}_{2} \text { respecto a } \\
\text { valores legislados } \\
\text { Inmisiones de partículas } \\
\text { respecto a valores legislados }\end{array}$ & $\begin{array}{l}\text { Gastos per cápita en medio } \\
\text { ambiente de ayuntamientos }\end{array}$ \\
\hline Pérdida de suelo & $\begin{array}{l}\text { Superficie total } \\
\text { incendiada }\end{array}$ & $\begin{array}{l}\text { Suelos con grave riesgo de } \\
\text { erosión }\end{array}$ & $\begin{array}{l}\text { Repoblación hidrológica- } \\
\text { forestal } \\
\text { Inversión en lucha contra la } \\
\text { erosión }\end{array}$ \\
\hline $\begin{array}{l}\text { Cantidad de } \\
\text { agua }\end{array}$ & $\begin{array}{l}\text { Intensidad de uso del } \\
\text { agua }\end{array}$ & $\begin{array}{l}\text { Sobreexplotación de acuíferos } \\
\text { Superficie con balance } \\
\text { hídrico deficitario } \\
\text { Recursos disponibles por } \\
\text { habitante }\end{array}$ & $\begin{array}{l}\text { Tasa de reducción de pérdidas } \\
\text { en conducciones } \\
\text { Tasa de variación del precio } \\
\text { del agua }\end{array}$ \\
\hline
\end{tabular}

Fuente. Monografía "Indicadores Ambientales. Una propuesta para España”. Ministerio de Medio Ambiente (1996). 
f) iiSBE (International Initiative for Sustainable Built Environment) promueve la adopción de políticas y métodos para el reconocimiento de la sostenibilidad en la construcción.

Algunos de los recursos expuestos anteriormente no presentan de forma explícita un conjunto estructurado de indicadores; no obstante, en todos ellos los resultados de los estudios se expresan por medio de indicadores de sostenibilidad.

Si se analizan los sistemas de indicadores propuestos por las diferentes entidades reseñadas, se comprueba que éstos no se ajustan completamente a los requerimientos propios de la actividad constructiva. No obstante, muchas de las compilaciones propuestas son útiles a nivel conceptual para realizar este análisis. Las particularidades del sistema constructivo y su contribución positiva y negativa al Desarrollo Sostenible requieren de sistemas de indicadores propios. Esto se ha puesto de manifiesto en las últimas décadas con la problemática planteada en el desarrollo de procedimientos de evaluación ambiental de edificios.

Entre los esfuerzos más notables producidos para establecer las bases de un conjunto de indicadores que permitan definir los aspectos que deben considerarse con objeto de promover la sostenibilidad en los edificios, destacan los trabajos desarrollados desde ISO (Organización Internacional para la Normalización), en concreto la serie ISO 14000, sobre gestión medioambiental. Actualmente, el comité ISO/TC 59/SC 17 ha desarrollado un documento, Sustainability in Building Construction - Sustainability Indicators. Part 1 Framework for the development of indicators for building, que tiene por objeto proporcionar una guía para el desarrollo y la selección de los indicadores de sostenibilidad relacionados con la construcción.

Este documento asume una estructura con justificación medioambiental como base de cualquier análisis del impacto que produce una determinada actividad sobre el medio ambiente (10). La estructura medioambiental proporciona un medio que describe las interacciones físicas entre cualquier sistema técnico y el medio que lo rodea, incluyendo en los dos sentidos flujos de energía, agua, materiales, residuos y emisiones (9).

Desde el punto de vista del impacto medioambiental, uno de los sistemas técnicos más significativo es el edificio, y sus infraestructuras asociadas (sistemas como el agua potable, agua consumida, residuos sólidos, transporte, comunicación y energía) ya que cada edificio, como cualquier sistema técni- co, interactúa con el medio ambiente. Estas interacciones físicas se producen desde el momento que se fabrican los productos que forman parte de los elementos constructivos hasta el proceso de demolición del edificio y el tratamiento de los residuos generados. Es decir, el edificio interactúa tanto con su ambiente interior como con el exterior a lo largo de todo su ciclo de vida.

Los métodos de evaluación del comportamiento medioambiental de los edificios deben:

- Proporcionar un conjunto común y verificable de criterios y metas de modo que los propietarios de edificios que defienden normas medioambientales más elevadas tengan un instrumento para medir, evaluar y demostrar dicho esfuerzo.

- Proporcionar una referencia que sirva de base común, mediante la cual los propietarios de edificios y los equipos de diseño puedan formular estrategias de diseño medioambientales que resulten efectivas.

- Recoger y organizar información detallada sobre el edificio que pueda utilizarse para reducir costes de explotación, financieros y seguros, reducir porcentajes de desocupación y aumentar la comerciabilidad.

- Predefinir el proceso de diseño mediante una declaración clara de lo que se considera que son aspectos medioambientales claves y sus prioridades relativas.

Los métodos de Análisis del Ciclo de Vida (ACV) juegan un papel importante a la hora de establecer criterios de comportamiento dentro de los métodos de evaluación ambiental global de los edificios. Sin embargo, al objeto de lograr las metas prácticas indicadas anteriormente es necesario que los métodos para evaluar el comportamiento medioambiental de los edificios seleccionen un número limitado de criterios y busquen el equilibrio entre el rigor y la practicidad; debido al gasto que supone recoger y mantener datos actuales de todos los elementos de un edificio.

Entre las herramientas de Análisis de Ciclo de Vida, destacan por su repercusión en medios de difusión científicos, las aplicaciones informáticas Gabi (IKP), LCAiT (Chalmers), SimaPro (Ecobilan Group) y Humberto (IFEU).

A partir de un inventario de las cargas ambientales, y haciendo uso de bases de datos de caracterización ambiental, estos programas determinan la medida cuantitativa del impacto negativo, es decir, los valores de cada categoría de impacto que describen el comportamiento medioambiental del producto. 
La precisión de estas aplicaciones informática es tal, que resulta inviable su aplicación para el análisis de un sistema tan complejo como un edificio completo. No obstante, sí resulta útil, como demuestran determinados trabajos de investigación en este sentido, en la evaluación ambiental de elementos más simples del edificio.

Además de los métodos de ACV, existe otro tipo de herramientas usadas en el análisis y evaluación de edificios que considera aspectos más amplios que las basadas en el $\mathrm{ACV}$, las denominadas 'Check List'. En ellas, se puede considerar que cada apartado puntuado constituye, en sí mismo, un indicador.

Estas herramientas evalúan cuestiones tan difícilmente computables como las relacionadas con el confort de los ocupantes o el control de la obra. No obstante, el grupo de indicadores propuestos y la forma de valorarlos no se ajustan del todo a un método de precisión matemática, quedando muchos aspectos al albedrío del técnico encargado de su valoración. Además, no trabajan con un sistema estructurado de indicadores.

Pertenecen a este tipo de herramientas sistemas tan conocidos como el LEED (Leadership in Energy and Environmental Design) o BREEAM (Building Research Establishment Environmental Assesment Method), o incluso la reciente GESV (Guía de Edificación Sostenible para la Vivienda, 2006), promovida por el Gobierno Vasco.

Tras el análisis de los sistemas de indicadores de mayor difusión, como se ha indicado anteriormente, se ha propuesto su agrupación, según su finalidad, en los siguientes grupos:

- Indicadores que tratan de determinar la contribución a un determinado impacto de materiales y productos a lo largo de todo su ciclo de vida. Los indicadores existentes en este grupo encajan en la metodología propuesta para un análisis de ciclo de vida del edificio. Se expresan en unidades cuantificables de forma matemática e inequívoca (p.e. emisiones de $\mathrm{kg} \mathrm{CO}_{2}$ eq, $\mathrm{m}^{3}$ de agua consumida, etc.), en los resultados generados por herramientas como Ecoquantum, Equer, etc. Estas herramientas presentan un conjunto de unos 15 indicadores, generalmente rígidos, admitiéndose pocas transformaciones y por lo tanto, poca capacidad de adecuación a hallazgos científicos, necesidades sociales, etc.

- Indicadores establecidos en herramientas que tratan de evaluar la sostenibilidad de los edificios desde un punto de vista global. Estas herramientas tratan de determinar los impactos producidos por el edificio a lo largo de toda su existencia; desde la fabricación de los productos que lo forman hasta su demolición, sin seguir la metodología propia del ACV. Aquí, se hace referencia a herramientas tipo 'Check List', como BREEAM o LEED, o a herramientas como CASBEE o SBTool. En este tipo de herramientas los indicadores son más numerosos, presentándose en conjuntos de unos 50 indicadores y abarcan, generalmente, una mayor variedad de aspectos que el grupo de indicadores reseñados anteriormente. A diferencia de aquellos, los sistemas de indicadores propuestos por estas herramientas presentan una importante flexibilidad, sufriendo transformaciones en función de las necesidades que se planteen en cada momento.

- Indicadores que ayudan a analizar la sostenibilidad a escala urbana o territorial. Se trata de amplias relaciones de indicadores (normalmente más de cien) que pretenden considerar todos los aspectos que resultan significativos al tratar de evaluar el estado actual y las tendencias existentes. Son los que han sufrido mayor desarrollo en los últimos años. Se estructuran, generalmente, de acuerdo a cada una de las áreas estudiadas en Desarrollo Sostenible. Muchos de los indicadores expresados en este tercer grupo pertenecen a sistemas de indicadores propuestos para el análisis de productos, sistemas, o edificios; otros, en cambio, no.

Los conjuntos de indicadores pertenecientes al grupo primero y segundo, son los que se están usando actualmente para diseñar, analizar y evaluar edificios desde el punto de vista de la sostenibilidad. En muy pocas ocasiones, las herramientas de diseño, análisis y evaluación consideran cuestiones sociales o económicas; ni siquiera, análisis rigurosos y precisos de aspectos como el confort, la integración del edificio en el entorno, etc. Se trata, en general, de variables y parámetros que describen la Eco-eficacia del sistema constructivo y no su Eco- Eficiencia (7).

En cualquier caso, todos estos sistemas pretenden alcanzar el objetivo de aproximación a un modelo de construcción que dé respuesta a las necesidades actuales, dentro del ámbito medioambiental, social y económico existente. Lo cual no ha encontrado todavía respuesta en los actuales documentos desarrollados para el análisis de edificios.

No obstante todo lo anterior, conviene apuntar aquí el previsible cambio conceptual de las herramientas de evaluación futuras, que pasarán de estructurarse en áreas, criterios y categorías, que requieren un consenso sobre justificaciones menos objetivas, para la ponderación final de indicadores. En su lugar, se 
sugiere considerar impactos y la valoración de su reducción en función de la aplicación de una determinada medida.

\section{APLICACIÓN AL CASO DE ESTUDIO: EL VALLE MINERO DE LACIANA (LEÓN)}

En el marco del proyecto de investigación del Plan Nacional I+D+i 2004-2007 "Modelo de construcción sostenible para la recuperación y protección de zonas medioambientalmente degradadas o frágiles: el Valle de Laciana" (BIA 2004-7654), se ha desarrollado un modelo de intervención para construcciones, infraestructuras y su entorno, desde criterios de sostenibilidad. En este artículo, se exponen los resultados alcanzados sobre la aplicabilidad de indicadores y las consideraciones a tener en cuenta en su selección para la evaluación de elementos en general, y de edificios en particular.

El interés de la aplicación de los indicadores a un elemento, varía en función del agente que solicite su estudio. Para los cuatro elementos finalmente seleccionados en el proyecto de investigación:

- Elemento E, edificaciones residenciales e instalaciones auxiliares

- Elemento R, lugar de depósito de residuos o vertidos/escombrera

- Elemento M, mina, lugar de explotación minera

- Elemento $\mathrm{CH}$, chabolo o conjunto de chabolos

Se enuncian los siguientes agentes implicados:

- Empresas, Promotores y propietarios de edificios y sitios

Minero-Siderúrgica de Ponferrada.

Los indicadores ayudarán a los promotores y propietarios de edificios a establecer los requerimientos relacionados con la sostenibilidad y sus objetivos. Los indicadores y métodos relacionados ayudarán a mostrar la conformidad del diseño o de la construcción con los requerimientos fijados. Los propietarios o evaluadores también pueden aplicar indicadores para mostrar y demostrar la sostenibilidad del elemento en el mercado.

- Proyectistas, Diseñadores, Investigadores

Universidad Politécnica de Madrid y Universidad de Sevilla, como instituciones implicadas en este proyecto de investigación; en segundo término, las universidades de Granada, CEU y London Metropolitan University, como implicadas en los Talleres y Cursos realizados; Universidad de Carlos III, a través de su Programa de Verano desarrollado en el Campus de Villablino; otros participantes afines a ellas.

Los indicadores ayudarán a diseñar identificando los parámetros esenciales relacionados con la sostenibilidad. Esto asegura que el diseñador es capaz de reconocer las características de diseño que tienen un efecto en los indicadores elegidos. Un diseñador también puede necesitar directrices sobre soluciones ventajosas en términos de recomendaciones prácticas. Dichas recomendaciones pueden demostrar las ventajas de sus diseños con la ayuda de los indicadores y los métodos de evaluación.

Con el uso de indicadores y los métodos y herramientas de evaluación correspondientes, pueden compararse diseños alternativos y puede demostrarse la conformidad de un diseño respecto a los objetivos establecidos.

\section{- Constructores}

Los constructores deberán estar informados de los requisitos relacionados con la sostenibilidad de los edificios en términos de indicadores. Además, los constructores pueden aplicar indicadores de sostenibilidad para realizar un seguimiento y control de los procesos de construcción.

- Administración: Junta de Castilla y León, Ayuntamiento de Villablino

La administración, posiblemente en línea con sus objetivos políticos, puede usar los indicadores para establecer y mostrar los requisitos relativos a la sostenibilidad de sitios y edificios. La administración puede establecer incentivos en relación a ciertas prestaciones o comportamientos de los edificios.

\section{- Usuarios}

Corporaciones locales del Valle de Laciana; Fundación Sierra-Pambley; Asociaciones, gremios y colectivos que previsiblemente participarán en las actuaciones vinculadas a la propuesta.

Los diferentes usuarios reflejarán mediante los indicadores las prestaciones y necesidades de infraestructuras y servicios que requieren.

- Gestores de mantenimiento del patrimonio

Los indicadores de sostenibilidad proporcionarán parámetros para realizar un seguimiento y control de la fase de uso de los edificios y sitios. 
Toda propuesta de indicador debe ir acompañada de su correspondiente procedimiento de validación; es decir, un indicador debe estar bien fundamentado, en términos técnicos y científicos. La Tabla 3 muestra las pautas para la selección y caracterización de indicadores.

También se perfilan las posibles restricciones que se pueden encontrar en el momento de su aplicación (véase Tabla 4).

\subsection{Propuesta de indicadores para la evaluación de elementos en el Valle minero de Laciana}

Para evaluar la situación y tendencia de los elementos y procesos del caso de estudio, se plantea aquí una propuesta de indicadores de forma que la metodología sea igualmente de aplicación en otros valles mineros de problemática similar, o territorios cuya fragilidad esté condicionada por la presencia de determinadas actividades económicas e instalaciones asociadas a ellas. Esto permitirá una visión global del territorio y se favorecerá la intervención integrada del conjunto. Todo ello queda recogido en la Tabla 5.

Con ello se pretende poder medir periódicamente el comportamiento sostenible de los elementos en los ámbitos considerados, tanto el entorno del valle, como el de las propias edificaciones (estado y comportamiento en la gestión de obra). Para hacer una selección de indicadores relevante es esencial identificar todas aquellas afecciones, impactos o puntos débiles más significativos que se producen en el caso de estudio, derivados de las actividades económicas. Esto incluye considerar la situación actual, además de las metas sociales y otras exigencias externas en el marco de la sostenibilidad. Basándose en esta información, se pueden definir los aspectos que requieren atención para el establecimiento y uso de indicadores (25).

El modelo de la propuesta está basado principalmente en dos dimensiones de análisis (27):

Tabla 3

Caracterización de un indicador para su cuantificación y disponibilidad de los valores requeridos (17).

\section{Un indicador deberá:}

Ser controlable.

- Estar fácilmente disponible a una relación razonable coste/beneficio.

- Estar adecuadamente documentado y ser de calidad reconocida.

- $\quad$ Actualizarse en concordancia con los procedimientos seguros.

- $\quad$ Tener un valor de comparación como referencia.

Fuente. Elaboración propia.

Tabla 4

Posibles restricciones en la aplicación de indicadores (17)

Posibles restricciones de un indicador:

- Diferente disponibilidad de datos e información en los ámbitos nacional e internacional.

- Falta de normativas metodológicas en el contexto internacional.

- Diferente ranking de indicadores en el ámbito internacional y por tanto, diferente tratamiento legal, que lleva a una falta de balance en la valoración internacional.

- Falta de niveles de referencia, figuras de base, de forma que no se puede proporcionar orientación sobre tendencias, por ejemplo, para la biodiversidad.

- Relación espacial que no se considere suficientemente. A escala internacional el uso de valores medios a nivel de país, predominan sobre los de nivel regional.

- $\quad$ Son necesarios programas de monitorización a largo plazo para cambios medioambientales a largo plazo.

- Dominan los parámetros cuantificables para la caracterización de un indicador, en detrimento de aquellos impactos de valor cualitativo, como por ejemplo, estéticos, éticos o culturales.

- $\quad$ Falta de modelos que hagan de puente ante esos déficits de información.

- Falta de prioridad para establecer indicadores, de forma que el rango de estrategias sostenibles no puede fundamentarse todavía en indicadores de Sostenibilidad.

- $\quad$ En algunos casos, es difícil obtener valores porque las unidades paisajísticas no coinciden con los límites o áreas administrativas.

- $\quad$ Se observa la falta de inventarios de elementos culturales. 


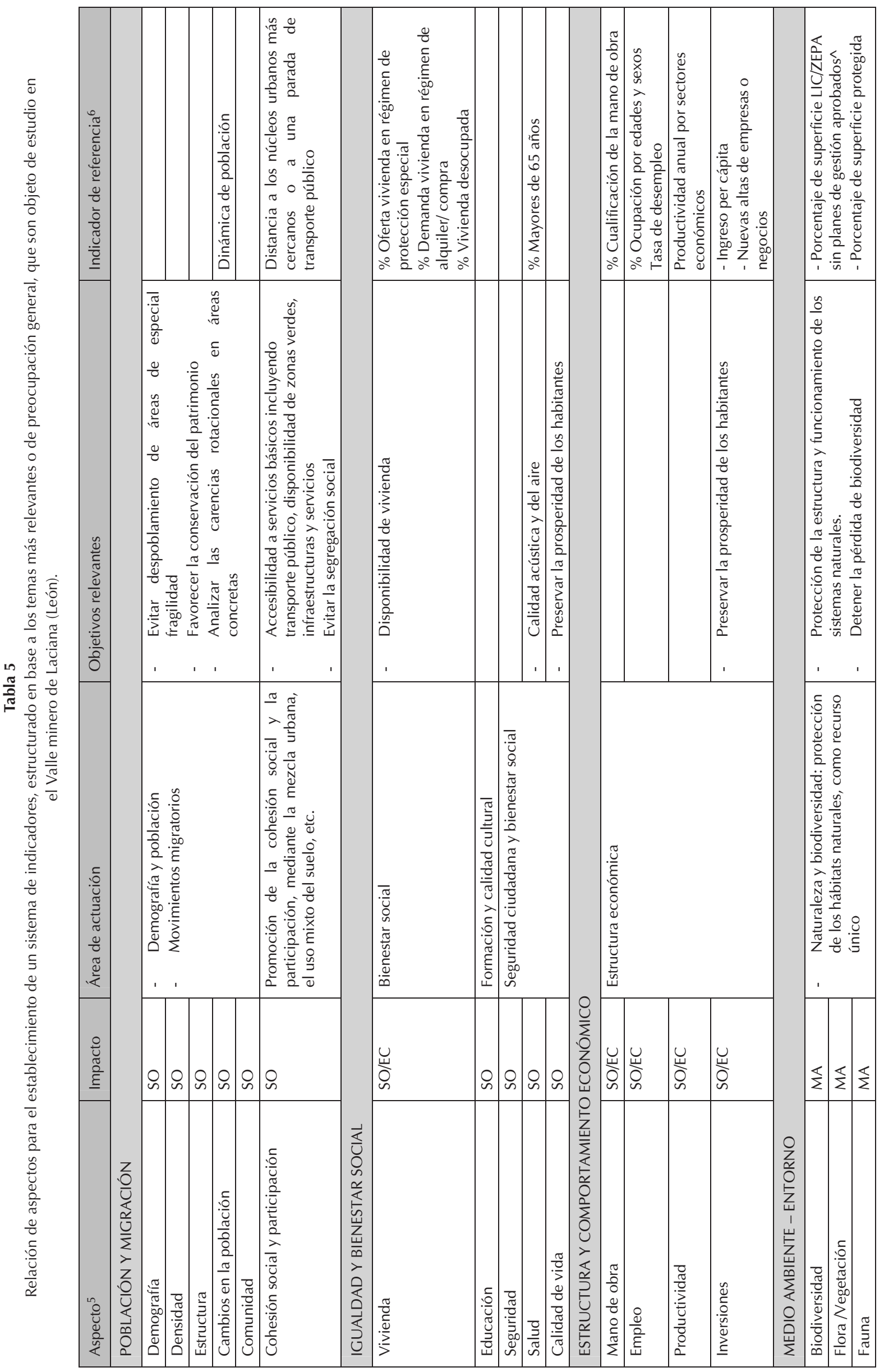




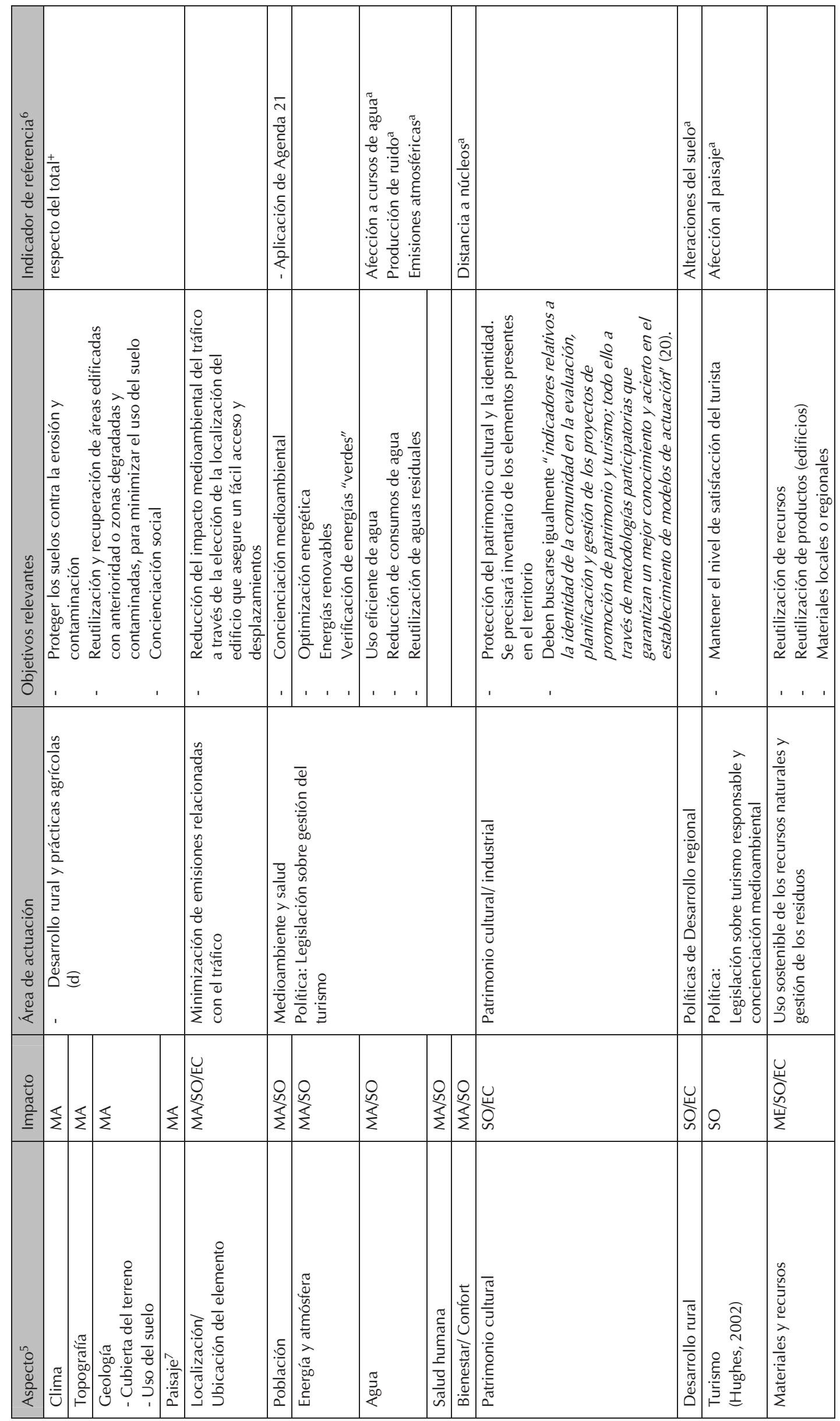




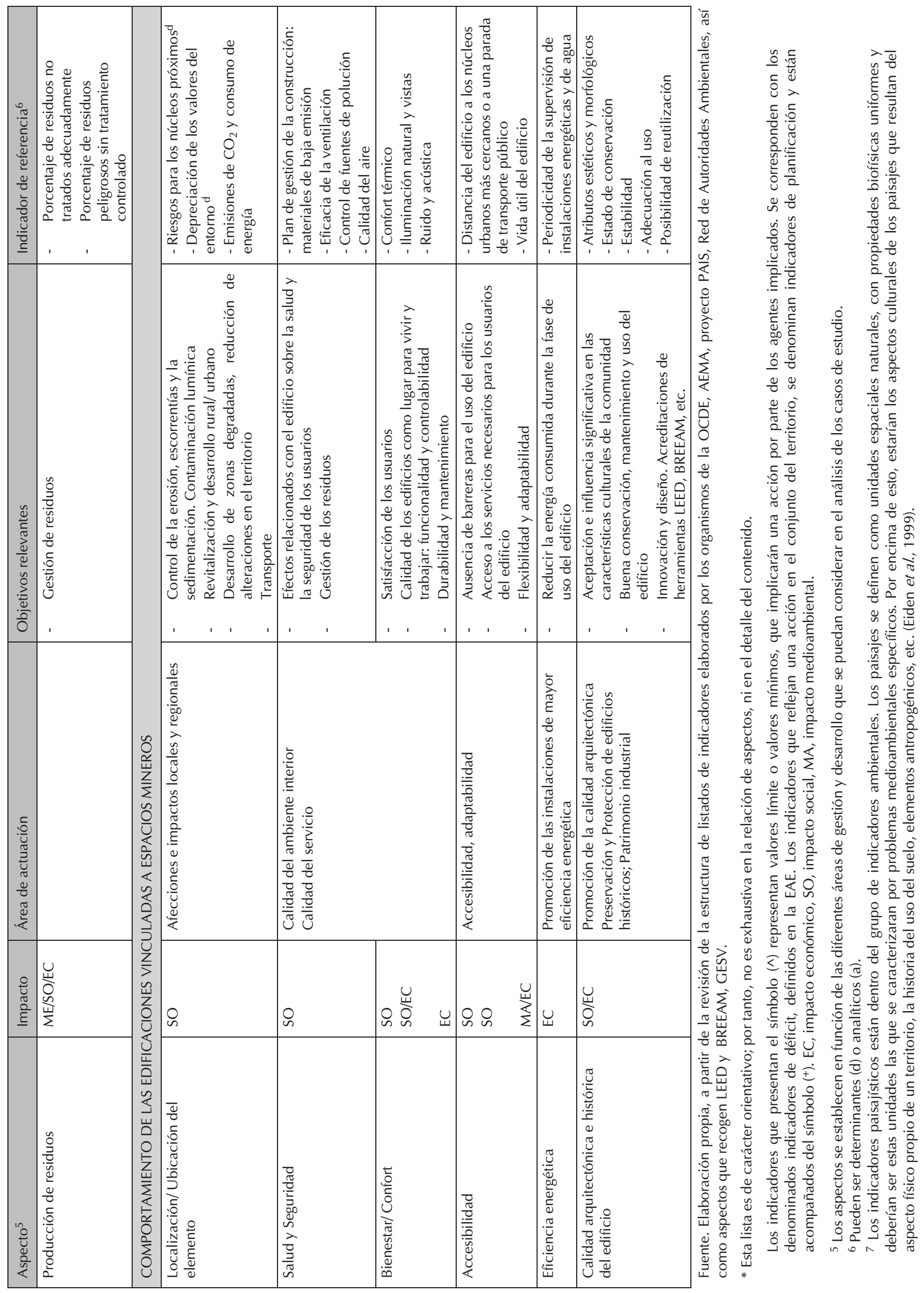


- Ámbito/ Tema (véase Tabla 5)

Esta dimensión define el contexto de aplicación de cada uno de los indicadores. En el modelo de propuesta se han considerado los siguientes:

o Población y migración

o Igualdad y bienestar social

o Estructura y composición económica

o Medioambiente - Entorno

o Comportamiento de las edificaciones vinculadas a espacios mineros

- Tipo de indicador (1)

Esta dimensión considera la tipología y definición de los indicadores que se consideran clave en este estudio para el comportamiento sostenible. Actualmente disponemos de una amplia relación de indicadores, tanto desde el punto de vista del análisis de las afecciones absolutas de elementos/ productos, como del análisis de territorios o entornos concretos, según lo expuesto anteriormente. Los aspectos analizados, se reflejan en los formularios del presente artículo, ya que han constituido una fuente de información conceptual básica de este trabajo.

Estos indicadores asumidos de forma convencional se han complementado con una serie de índices que dan respuesta a la especificidad territorial y al espacio natural y urbano, deteriorado por la acción antrópica. Los parámetros seleccionados determinan no solamente la afección ambiental de elementos y procesos, principalmente la actividad minera, sino que también constituyen los referentes para un análisis global del entorno del valle.

Por ello, en la propuesta se va a diferenciar entre:

- Factores/ indicadores que recomienden una actuación enérgica o trascendental sobre el territorio. Esta acción se producirá tanto en positivo como negativo, es decir, tanto para conservar como para eliminar el elemento objeto de estudio. A estos indicadores que tienen la capacidad de excluir actividades o elementos generados por la acción del ser humano se les ha denominado indicadores determinantes. Son, por lo tanto, aquellos parámetros que permiten decidir sobre la necesidad de mantener o no el resultado de esa acción antrópica, ya sea un edificio, escombrera o una instalación industrial.

- El segundo conjunto de indicadores, indicadores analíticos, lo constituyen las variables sintéticas que van a permitir obtener una visión holística de las líneas principales que se deben desarrollar en la futura actuación. La cuantificación de estos indicadores tiene sentido realizarla en aquellos casos en los que, de la aplicación de los indicadores excluyentes, resulte la conveniencia de protección, conservación, reforma, etc. de un elemento. Debido a este sentido de globalidad, tendrán tanto un carácter intrínseco como extrínseco, es decir, tanto de la acción antrópica en relación a su entorno, como en sí misma.

Como se ha señalado anteriormente, el indicador debe identificarse con un valor cuantitativo (auque sea un indicador cualitativo), su expresión debe permitir una cuantificación numérica inequívoca y determinante. En el caso de corresponder a fenómenos simples afectados por una única variable esta cuantificación será directa. A estos parámetros se les ha denominado indicadores simples. Estos indicadores no necesitan una expresión que relacione variables entre sí, ya que según la definición dada, solo depende de una única variable; por ejemplo, el indicador 'Distancia a núcleos de población próximo' es una variable única, la longitud de la línea que une el elemento analizado con el núcleo de población más próximo.

No obstante, la mayoría de los indicadores propuestos son el resultado de la consideración de dos o más variables, son indicadores complejos. La valoración de estos indicadores resulta, lógicamente, de una expresión matemática más compleja. Por ejemplo, el indicador 'Alteración del suelo' depende del tipo de alteración y de la potencialidad de uso del suelo.

En cualquier caso, cada una de las variables consideradas para un indicador, podrá estar afectada por un coeficiente de carácter técnico o coeficiente corrector, que introduce el factor que mide la importancia o contribución de esa variable respecto a la totalidad de la acción.

\subsubsection{Aproximación a los indicadores deter- minantes}

Dada la naturaleza de estos indicadores, son siempre parámetros extrínsecos, dado que no dependen de la actividad en sí misma sino, más bien, del efecto que esta produce en su entorno inmediato, próximo, o lejano. En el caso de estudio, los más significativos son los siguientes:

\section{I.1. Riesgos para los núcleos próximos (R).}

Consecuencias de riesgo para la salud o seguridad, de las personas o colectivos. Su valor es más alto cuanto mayor sea el riesgo. En la definición numérica de este indicador intervienen múltiples factores, se trata, pues, 
de un indicador complejo. Las variables fundamentales que intervienen en su cuantificación son:

a) El riesgo de desprendimiento (p.e. ángulos pronunciados de talud en depósitos antrópicos)

b) El riesgo de deslizamientos (p.e. el caso edificaciones situadas en laderas)

c) El riesgo de hundimientos (p.e. sobrecargas excesivas en planos de apoyo)

d) La generación de suciedad y contaminantes (p.e. polvo, smog, contaminantes)

e) La existencia de problemas sanitarios (p.e. emisión de sustancias tóxicas)

La expresión matemática que relaciona estas variables es:

$\mathrm{R}=\mathrm{De} \cdot \mathrm{a}_{\mathrm{r}-1}+\mathrm{Ds} \cdot \mathrm{a}_{\mathrm{r}-2}+\mathrm{Hu} \cdot \mathrm{a}_{\mathrm{r}-3}+\mathrm{Su} \cdot \mathrm{a}_{\mathrm{r}-4}+\mathrm{Sn} \cdot \mathrm{a}_{\mathrm{r}-5}$

Siendo:

De: Desprendimientos (valorado en una escala de 0 a 3)

Ds: Deslizamientos (valorado en una escala de 0 a 3 )

$\mathrm{Hu}$ : Hundimientos (valorado en una escala de 0 a 3)

Su: Suciedad y contaminantes (valorado en una escala de 0 a 3 )

Sn: Problemas sanitarios (valorado en una escala de 0 a 3)

ar-i: Coeficiente corrector.

I.2. Depreciación de los valores del entorno (D).

Determina el grado de afección que la actividad analizada produce en su entorno. Es mayor cuanto más se afecte de forma negativa el entorno, pudiendo resultar un valor negativo en aquellos casos que se generen, restauren o potencien los valores del entorno. Resultará un valor más alto, cuando mayores sean los valores de éste, considerándose los siguientes:

a) Patrimoniales o culturales

b) Valores ecológicos endémicos

La expresión matemática que cuantifica este indicador es la siguiente:

$\mathrm{D}=$ Pe $\cdot$ Ape $\cdot \mathrm{a}_{\mathrm{d}-1}+\mathrm{Cu} \cdot \mathrm{Acu} \cdot \mathrm{a}_{\mathrm{d}-2}+\mathrm{Ec} \cdot$ Aec $\cdot \mathrm{a}_{\mathrm{d}-3}$

Siendo:

Pe: Existencia de valores patrimoniales (valorado en una escala de 0 a 3 )

Cu: Existencia de valores culturales (valorado en una escala de 0 a 3)

Ec: Existencia de valores ecológicos endémicos (valorado en una escala de 0 a 3 ) Ape: Grado de afección sobre valores patrimoniales (valorado en una escala de -3 a 3)
Acu: Grado de afección sobre los valores culturales (valorado en una escala de -3 a 3) Aec: Grado de afección sobre valores ecológicos (valorado en una escala de -3 a 3 )

Con la cuantificación de estos dos indicadores es posible obtener una valoración única que nos permita decidir la exclusión del elemento analizado. Esta valoración única será el resultado de considerar ambos valores, afectados cada uno de ellos por un coeficiente. Este coeficiente y la regla de corte se establecerán a priori según las necesidades y expectativas del grupo humano encargado de gestionar el medio ambiente afectado.

Si se mide en un baremo de 0 a 10, esta valoración única $(\mathrm{V})$ deriva en la siguiente expresión matemática:

$V=\left(R b_{1}+D b_{2}\right) \cdot\left[10 /\left(R_{\max } b_{1}+D_{\text {max }} b_{2}\right)\right]$

Siendo $b_{\mathrm{i}}$ :

Coeficiente corrector socioeconómico, mide la importancia dada a cada afección.

\subsubsection{Aproximación a los indicadores analíticos}

Miden tanto las cualidades extrínsecas como intrínsecas del elemento o actividad analizada, marcando la dirección a seguir en la toma de decisiones para la conservación o restauración del aspecto o entorno afectado.

II.1. Indicadores globales intrínsecos. Miden cualidades propias del elemento. Son indicadores simples y, por lo tanto, no precisan coeficientes correctores ni formulación de variables. Evaluarán al menos las siguientes características:
a) Atributos estéticos y morfológicos
b) Estado de conservación
c) Estabilidad
d) Adecuación al uso
e) Posibilidad de reutilización

II.2. Indicadores globales extrínsecos. Miden la relación entre el elemento y el entorno. Evalúan las consecuencias ambientales de una acción antrópica.

II.2.1. Indicadores físicos. Miden el grado de afección sobre el entorno físico. Dentro de esta categoría podemos distinguir los siguientes:

a) Afección paisajística (P). Determina en qué medida se ven afectados, desde el punto de vista de la percepción (visual, sonora, olfativa etc.), los valores del paisaje natural circundante a la actividad o elemento anali- 
zado. Se trata de un indicador complejo que es función de las siguientes variables:

$P=V g a_{p-1}+A m a_{p-2}+V_{s a} a_{p-3}+M a_{p-4}$

Siendo:

Vg: Afección a la vegetación (valorado en una escala de 0 a 3 )

Am: Alteración morfológica (valorado en una escala de 0 a 3)

Vs: Afección visual (valorado en una escala de 0 a 3)

Mr: Modificación del relieve (valorado en una escala de 0 a 3 )

$\mathrm{a}_{\mathrm{pi}}$ : Coeficiente corrector.

b) Alteración del suelo (S). Indicador complejo que evalúa el grado de afección de la actividad estudiada sobre la capa superficial del terreno. A su vez, esta capa del terreno será función del uso potencial o previo del suelo (agrícola, forestal, etc.), o del tipo de alteración que presenta (p.e. pérdida de la capa de tierra vegetal productiva, emisión de contaminantes al suelo, etc.). A cada una de estas variables, y en función de su naturaleza se otorgará un valor numérico.

$\mathrm{S}=\left(\mathrm{Al} \mathrm{a}_{\mathrm{s}}\right) \cdot(\mathrm{Po})$

Siendo:

Al: Tipo de alteración (en función del tipo se valorará de 1 en el caso de alteraciones leves a 5 en el caso de alteraciones graves) Po: Potencialidad / uso original (se valorará de 1 a 3 en función del valor del uso posible del suelo)

$\mathrm{a}_{\mathrm{s}}$ : Coeficiente corrector

c) Afección a cursos de aguas (W). Nos da una idea de en qué medida la actividad objeto de estudio altera los cursos naturales o artificiales de agua. La cuantificación de este indicador depende de varios factores, el valor numérico será mayor cuanto mayor sea la afección.

$W=R d a_{w-1}+S s a_{w-2}+L x a_{w-3}+E n a_{w-4}$

Donde:

Rd: Alteración de la red de drenaje (valorado en una escala de 0 a 3)

Ss: Aumento de sólidos en suspensión (valorado en una escala de 0 a 3 )

Lx: Incorporación de lixiviados (valorado en una escala de 0 a 3 )

En: Aparición de encharcamiento (valorado en una escala de 0 a 3)

$\mathrm{a}_{\mathrm{w}-\mathrm{i}}$ : Coeficiente corrector.

II.2.2. Indicadores sociales, culturales y económicos. Son indicadores que miden el gra- do de afección sobre la población próxima a la actividad o elemento.

a) Producción de ruido (R). Es un indicador simple que cuantifica el nivel de ruido y que provoca la actividad o elemento estudiado. Donde R tiene un valor de:

0, si el ambiente acústico es bajo (<40 dbA)

1, si el ambiente acústico es medio (entre $40 \mathrm{dbA}-60 \mathrm{dbA}$ )

2, si el ambiente acústico es alto (> 60 dbA)

b) Emisiones atmosféricas (E). Indica la capacidad de emisión gases, olores o partículas a la atmósfera. El valor de este indicador será más alto en la medida que la dirección y sentido del viento coincida con la de núcleos próximos.

$\mathrm{E}=\left(\Sigma A \mathrm{t}_{\mathrm{i}} \cdot \mathrm{N}_{\mathrm{i}}\right) \cdot \mathrm{V}$

Donde:

At: Valor en función del tipo de emisión (entre 1 y 5, mayor cuanto más insalubre, peligrosa o molesta. En este caso el rango es mayor ya que variedad del tipo de emisiones posible exige de una gradación mayor);

$\mathrm{N}$ : Valor en función de la cantidad emitida en la relación a una unidad de tiempo. En función del tipo de actividad;

$\checkmark$ : Nivel de coincidencia de la dirección y sentido de los vientos dominantes en la zona (se valora entre 1 y 4; según en el cuadrante que se encuentre en relación con la dirección y sentido del viento dominante).

c) Distancia a núcleos (L). Distancia medida en línea recta desde el elemento estudiado al núcleo de población más próximo medido en kilómetros. Por lo tanto, es un indicador simple con un cometido importante a la hora de determinar el impacto de la actividad o elemento sobre la población.

\subsection{Ejemplo de aplicación del sistema de indicadores propuesto}

Se ha tomado el elemento M4, Conjunto de extracción de carbón de Bolsada, como ejemplo para la aplicación del sistema de indicadores propuesto (véase Fig. 1, a y b).

Este elemento destaca por sus características especiales, tanto desde el punto de vista constructivo, como de su relación con el entorno.

Resulta, por tanto, adecuado para mostrar de forma clara el proceso de evaluación planteado, ya que su complejidad le hace representativo de las situaciones comunes a 

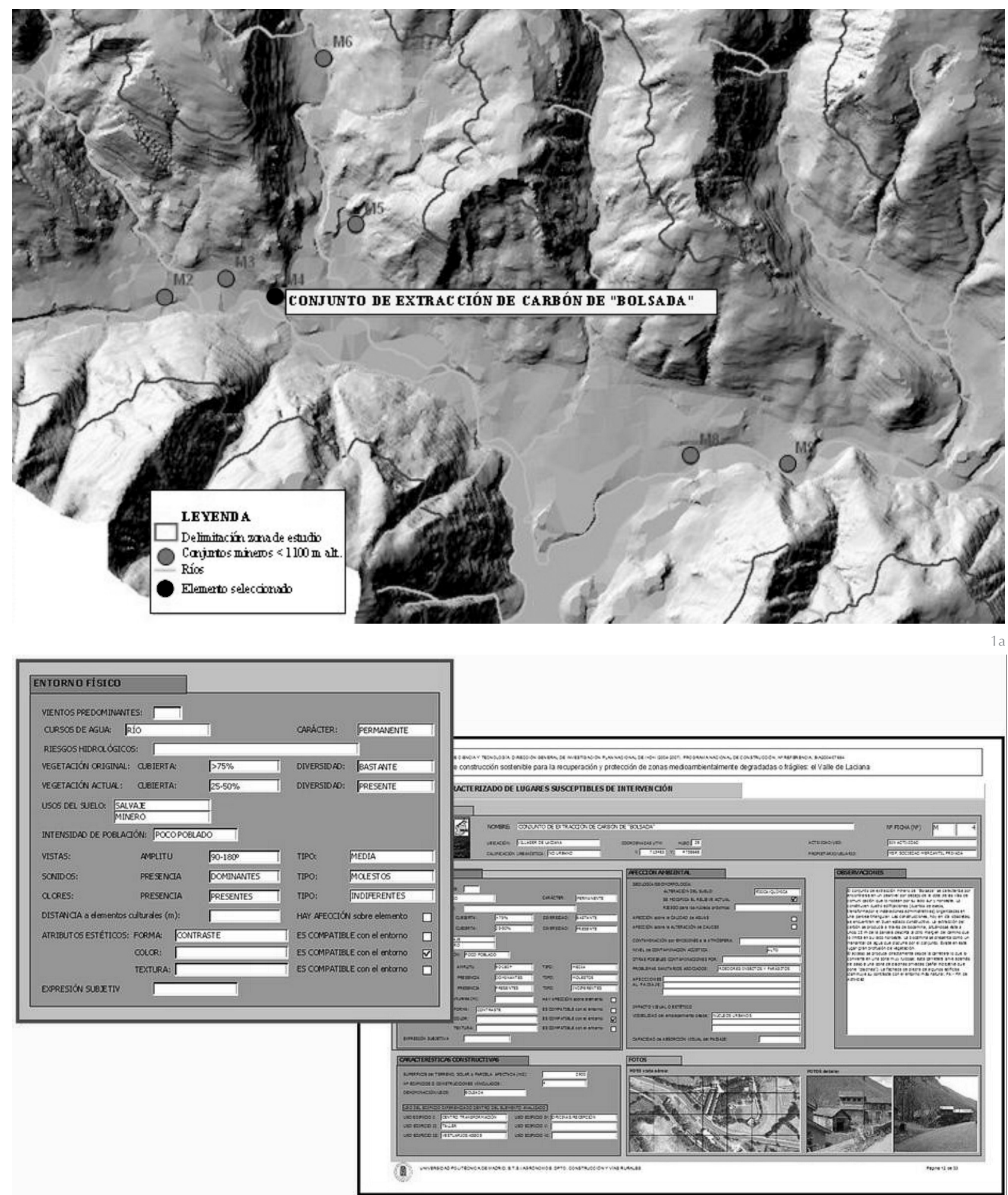

otros elementos de análisis en este tipo de territorios.

Es necesario aclarar que los valores asignados en esta aplicación a los coeficientes correctores de carácter técnico (a), se han fijado como una primera aproximación y serían los mismos en el análisis de todos los elementos a estudiar.
No obstante, el valor dado a los coeficientes correctores de carácter socio-económico (b) tiene una función meramente descriptiva del procedimiento; estos coeficientes deben determinarlos, como se ha dicho anteriormente, los responsables de la gestión medioambiental del territorio y, en ningún caso, los técnicos que realizan el análisis propiamente dicho.
1. a) Visualización simultánea en SIC de la ventana del área geográfica de estudio y de la edición del elemento M4 en la bdD, según leyenda adjunta; b) Informe del elemento M4 Conjunto de extracción de carbón de Bolsada., extraído de la base de datos generada en ACCESS que se incluye en SIG. 
4.2.1. Obtención de los valores de los indicadores determinantes

La evaluación de estos indicadores mostrará la conveniencia de conservar o no la existencia el elemento analizado. El objetivo principal del cálculo es determinar una valoración conjunta de los riesgos que genera el elemento y la depreciación de los valores existentes que produce. Si el valor obtenido es superior a un valor límite determinado, se recomendaría la exclusión del elemento y la restitución del estado original previo a su implantación. En nuestro caso, y al objeto de poder aplicar el procedimiento, se tomará como valor límite 5,00.

Buscando una mayor claridad, se omiten los cálculos intermedios de cada uno de los indicadores, aportándose los resultados finales en el resumen de la Tabla 6.

I.1. Riesgos para los núcleos próximos

I.2. Depreciación de los valores del entorno (D).

El valor de [2] $\mathrm{D}=0,85$ (en el caso extremo, el valor sería 20,70$)$.

Resultaría por tanto la valoración única siguiente, aplicada al ejemplo particular: [3] $V=0,268<5,00$. Se han tomado los siguientes valores de coeficientes correctores socioeconómicos: $b_{1}=0,6$ y $b_{2}=0,4$.

Como se puede comprobar, es una puntuación relativamente baja, considerando que el rango de esta valoración está comprendido entre 0 y 10 . Como es obvio, este resultado mide conjuntamente los dos indicadores determinantes propuestos. La puntuación obtenida indica que el elemento analizado no supone riesgo alguno para los núcleos próximos y que su existencia tiene una mínima repercusión sobre los valores del entorno. Dado que es inferior a 5,00, no se recomendaría la eliminación del elemento.

\subsubsection{Evaluación de los indicadores analíti-} $\cos$

II.1. Indicadores globales intrínsecos

II.2. Indicadores globales extrínsecos

II.2.1. Indicadores globales extrínsecos físi$\cos$
a. Afección paisajística. (P)
b. Alteración del suelo (S)
c. Afección a cursos de aguas (W)

II.2.2. Indicadores globales externalizables sociales, culturales y económicos II.3.a. Producción de ruido (R)

En nuestro caso y dado que el nivel de emisión de ruido es muy baja, inferior en cualquier caso a $40 \mathrm{dbA}, \mathrm{R}=0$

\section{II.3.b. Emisiones atmosférica (E) \\ II.3.c. Distancia a núcleos (L)}

En el caso del ejemplo propuesto, la distancia al núcleo de población más cercano, Caboalles de Abajo, es 1,08 km.

Tabla 6

Tabla resumen de los valores obtenidos para los indicadotres seleccionados, tras el análisis del ejemplo práctico, M04 Conjunto de extracción de carbón de Bolsada

\begin{tabular}{|c|c|c|c|c|}
\hline \multicolumn{2}{|r|}{ INDICADOR } & \multicolumn{2}{|c|}{ VALOR } & RANGO \\
\hline \multirow[b]{3}{*}{ Determinantes } & Riesgos para los núcleos próximos & $\mathrm{R}$ & 0,00 & $0,00-7,35$ \\
\hline & Depreciación de los valores del entorno & $\mathrm{D}$ & 0,85 & $-20,80-+20,70$ \\
\hline & $\begin{array}{c}\text { VALORACIÓN: } \\
0,268<5,00 \quad \text { NOEXCLUSIÓN }\end{array}$ & & & \\
\hline \multirow{5}{*}{ Analíticos Int. } & Atributos estéticos y morfológicos & Ila & 4,50 & $0,00-10,0$ \\
\hline & Estado de conservación & $\mathrm{Ilb}$ & 6,00 & $0,00-10,0$ \\
\hline & Estabilidad & Ilc & 8,50 & $0,00-10,0$ \\
\hline & Adecuación al uso & Ild & 8,50 & $0,00-10,0$ \\
\hline & Posibilidad de reutilización & Ile & 9,50 & $0,00-10,0$ \\
\hline \multirow{6}{*}{ Analíticos Ext. } & Afección paisajística & $\mathrm{P}$ & 3,88 & $0,00-8,85$ \\
\hline & Alteración del suelo & $\mathrm{S}$ & 1,50 & $0,00-15,00$ \\
\hline & Afección a cursos de aguas & W & 6,95 & $0,00-8,40$ \\
\hline & Producción de ruido & $\mathrm{R}$ & 0,00 & $0,00-3,00$ \\
\hline & Emisiones atmosférica & $\mathrm{E}$ & 0,00 & - \\
\hline & Distancia a núcleos & $\mathrm{L}$ & 1,08 & - \\
\hline
\end{tabular}


De los resultados mostrados en la Tabla 6, se pueden extraer las siguientes consideraciones:

- El elemento M4 no supone un riesgo significativo para los núcleos de población próximos.

- No se deriva de su existencia una depreciación de los valores existentes en el territorio. Aunque este aspecto se podría mejorar mediante la potenciación de valores ecológicos endémicos o mediante la generación de valores culturales o incluso patrimoniales (patrimonio industrial).

- Dada esas dos consideraciones y de acuerdo a la valoración conjunta de estos parámetros fundamentales, se determina la conveniencia de su conservación, rehabilitación o reforma.

- Posee atributos estéticos adecuados, un estado constructivo bueno y adecuadas condiciones de estabilidad estructural. Por lo tanto, la intervención sobre el elemento no incidirá de forma importante en aspectos constructivos.

- La afección paisajística se produce, principalmente, por la alteración de la topografía inicial y por la exposición del elemento M4 a líneas de visión importantes. Debido a la dificultad de incidir sobre el primer aspecto citado, y dado que éste puede resultar positivo en un planteamiento global, se prestará especial atención a la afección visual. La intervención reducirá en lo posible el impacto visual mediante estrategias de segregación, integración u ocultación.

- La producción de ruidos o emisiones es inexistente, por lo que no se tomará ninguna medida en este sentido.

- La existencia del elemento M4 provoca una alteración en la red de drenaje natural y en los cursos existentes, que origina la presencia de abundante agua procedente del subsuelo, produciéndose encharcamientos en todo el conjunto y su entorno. La actuación sobre este elemento debe, por lo tanto, reestructurar el flujo de agua natural existente.

\section{CONCLUSIONES}

Como se ha venido explicando detalladamente, se ha desarrollado una lista de trabajo de indicadores generales para medir o evaluar diferentes aspectos relacionados con la sostenibilidad de las construcciones y del paisaje de Laciana.

No obstante, es una lista flexible, que puede completarse o modificarse acorde con las prioridades, problemas y objetivos concretos de los agentes implicados, y de los modelos a evaluar.

Sin embargo, no es objeto del presente proyecto ni el establecimiento de una metodología para la monitorización de cada indicador propuesto ni la validación del procedimiento, lo que serviría de base para la justificación de su obtención, reconocimiento y respaldo para su uso en otras intervenciones en ámbitos similares (2). Esto constituye, en sí mismo, un trabajo de investigación adicional, que queda fuera de los objetivos establecidos originalmente.

Respecto del análisis de los resultados de la propuesta de indicadores para el caso de estudio, se ha constatado que la mayoría son de naturaleza compleja. Esto quiere decir, de acuerdo con la definición expuesta, que dependen de varias variables. La relación entre cada una de estas variables y su influencia sobre el resultado global la determina lo que hemos llamado un 'coeficiente corrector técnico a'. En el sistema de indicadores propuestos intervienen, además, unos 'coeficientes correctores socioeconómicos b', que establecen importancia relativa de los indicadores determinantes entre sí. La expresión numérica de cada uno estos coeficientes Correctores debe derivar de un análisis previo de las circunstancias del territorio y deben fijarse, lógicamente, a priori.

El sistema de parámetros planteado propone la valoración de cada una de las variables intervinientes. Esta valoración pretende ser el resultado directo de un análisis que precise poca instrumentación, a la vez que suficientemente certero para que sea descriptivo de las circunstancias reales del elemento de análisis. El equilibrio entre uno y otro aspecto, nos lleva a descomponer el fenómeno en múltiples factores simples. Cada uno de estos factores puede ser evaluado por un técnico cualificado de forma rápida y sencilla debido a la simpleza de la gradación propuesta.

No obstante, para evitar la introducción de componentes subjetivas en esta fase de la evaluación, será necesario llevar a cabo una revisión crítica de los valores asignados a cada factor.

En nuestro caso, el método de análisis empleado para la evaluación de indicadores se ha llevado a cabo en dos fases distintas. Por un lado, se ha elaborado un formulario para cada tipo de acción antrópica significativa en el territorio. En estos formularios se propone la valoración de cada factor interviniente en el sistema de indicadores. Por otro, para cada tipo de elemento de análisis 
se ha asignado un valor a cada factor establecido. Esta tarea de valoración de factores se ha llevado a cabo mediante un trabajo de campo complementado con un trabajo de gabinete.

De esta forma se ha caracterizado, de forma específica las actividades y elementos existentes en el territorio, caracterización que permite el necesario análisis previo a cualquier intervención posible.

\section{AGRADECIMIENTOS}

La investigación desarrollada en el presente artículo forma parte del proyecto de investigación "Modelo de Construcción Sostenible para la recuperación y protección de zonas medioambientalmente degradadas o frágiles: El Valle de Laciana", financiado por el Ministerio de Educación y Ciencia, en el marco del Plan Nacional de I+D+i 2004-2007 (referencia BIA 2004-07654), España.

\section{BIBLIOGRAFÍA}

(1) Agencia de Ecología Urbana de Barcelona (2007). Avance del Plan Especial de Indicadores de Sostenibilidad Ambiental de la Actividad Urbanística de Sevilla. Gerencia de Urbanismo. Ayto. Sevilla.

(2) Bookstaller, C. y Girardin, P. (2003). How to validate environmental indicators. Agricultural systems, 76: 639-653.

(3) Button, K. (2002). City management and urban environmental indicators. Ecological Economics, 40: 217-233. ESPECIAL SECTION: ECONOMICS OF URBAN SUSTAINABILITY.

(4) Eiden, G., Lucas, S., Piorr, H.-P., Stott, A., Blom, G., Fjellstad, W., Fais, A. (1999). Landscape indicators. OECD-Room Document, 3. OECD Expert Meeting on Biodiversity. Wildlife Habitat \&Landscape, París.

(5) Fernández-Latorre, F.M. (2006). Indicadores de sostenibilidad y medio ambiente: métodos y escala. Consejería de Medio Ambiente. Junta de Andalucía.

(6) Gallopín, G.C. (1997). Indicators and Their Use: Information for Decision-making. Moldan, B. and S. Bilharz Eds. SCOPE 58. Sustainability Indicators. A Report on the Project on Indicators of Sustainable Development. Wiley, Chichester.

(7) Huete, R. (2001). Aproximación a un Modelo de Construcción Ecoeficiente. Naturaleza, Cultura y Tecnología para un desarrollo urbano y territorial. Universidad de Sevilla. [http://tecnologiaedu.us.es]

(8) Hughes, G. (2002). Environmental indicators. Annals of Tourism Research, 29 (2): 457-477.

(9) IEA (2001). Environmental Framework. Annex 31 Energy-Related Environmental Impact of Buildings.

(10) ISO 14041 (1998). Environmental management-Life cycle assessment-Goal and scope definition and inventory analysis.

(11) ISO 14042 (2000). Environmental management-Life cycle assessment-Life cycle impact assessment.

(12) ISO 14050 (2002). Environmental Management-Vocabulary.

(13) ISO/ TS 21929-1 (2006). Sustainability in building construction-Sustainability indicators-Part 1: Framework for the development of indicators for buildings.

(14) LEY 9/2006, de 28 de abril, sobre evaluación de los efectos de determinados planes y programas en el medio ambiente.

(15) MMA, 2004. Evaluación ambiental de la Programación 2007-2013. Guía para los responsables de la planificación. Ministerio de Medio Ambiente. FEDER.

(16) Nicholson, M. y Fryer, R. (2002). Developing effective environmental indicators-Does a new dog need old tricks? Marine Pollution Bulletin, 45: 53-61.

(17) Piorr, Hans-Peter (2003). Environmental policy, agri-environmental indicators and landscape indicators. Agriculture, Ecosystems and Environment, 98: 17-33.

(18) Rayen Quiroga, M. (2001). Indicadores de sostenibilidad ambiental y de desarrollo sostenible: estado del arte y perpectivas. División de Medio Ambiente y Asentamientos Humanos. Santiago de Chile.

(19) Rueda, S. (1999). Modelos e indicadores para ciudades más sostenibles. Departamento de Medi Ambient. Generalitat de Catalunya. Agencia Europea de Medio Ambiente.

(20) Ruiz Ballesteros, E. \& Hernández Ramírez, M. (2007). Identity and community-Reflections on the development of mining heritage tourism in Southern Spain. Tourism management, 28 (3): 677-687.

(21) Smeets, E. y Weterings, R. (1999). Environmental Indicators: Typology and overview. European Environmental Agency. Tecnhical Report no 25.

(22) Stain, A., Riley, J., Halberg, H. (2001). Issues of scale for environmental indicators. Agriculture, Ecosystems and Environment, 87: 215-232.

(23) AEMA: El texto completo del informe Señales medioambientales 2041 puede consultarse en el sitio web de la AEMA, en la siguiente dirección [http://reports.eea.europa.eu/signals-2004/es/].

(24) GuíaEAE: [http://www.mma.es/secciones/raa/programacion_raa/eval_amb_raa/pdf/guiaeae_ fe_20072013es.pdf].

(25) Guía de indicadores medioambientales para la empresa (1999). IHOBE - BMU/ UBA. [http://www.ecoiurislapagina.com/biblio/dinteres/pdf/indicavasc.pdf].

(26) IISD (Internacional Institute for Sustainable Development): [http://www.iisd.org/measure/compendium/].

(27) NECSO: [http://www.necso.es/necso_esp/empresa/memeoria_513.asp].

(28) PNUMA: UNEP [http://www.unep.org/geo2000].

(29) WRI: [http://www.wri.org] 